Document downloaded from:

http://hdl.handle.net/10251/79776

This paper must be cited as:

Marton Lluch, I.; Martorell Aigües, P.; Mullor, R.; Sánchez Galdón, Al.; Martorell Alsina, SS. (2016). Optimization of test and maintenance of ageing components consisting of multiple items and addressing effectiveness. Reliability Engineering and System Safety. 153:151158. doi:10.1016/j.ress.2016.04.015

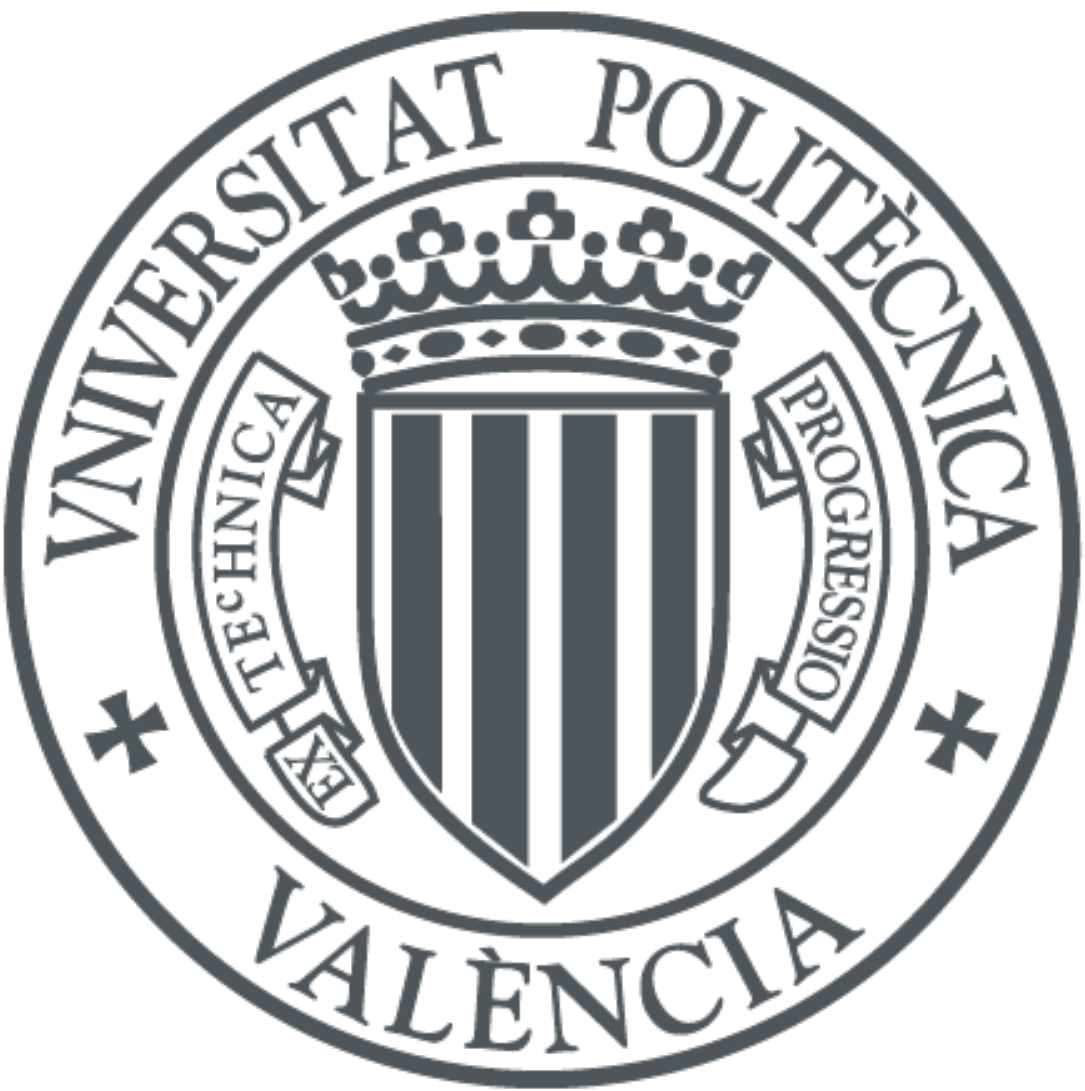

The final publication is available at

http://dx.doi.org/10.1016/j.ress.2016.04.015

Copyright Elsevier

Additional Information 


\section{Author's Accepted Manuscript}

Optimization of test and maintenance of ageing components consisting of multiple items and addressing effectiveness

I. Martón, P. Martorell, R. Mullor, A.I. Sánchez, S. Martorell

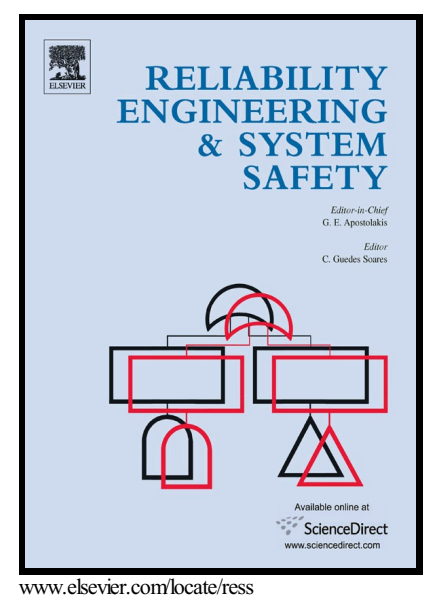

PII: $\quad$ S0951-8320(16)30043-6

DOI: $\quad$ http://dx.doi.org/10.1016/j.ress.2016.04.015

Reference: RESS5548

To appear in: Reliability Engineering and System Safety

Received date: 28 October 2015

Revised date: 5 April 2016

Accepted date: 28 April 2016

Cite this article as: I. Martón, P. Martorell, R. Mullor, A.I. Sánchez and S Martorell, Optimization of test and maintenance of ageing components consistin: of multiple items and addressing effectiveness, Reliability Engineering an System Safety, http://dx.doi.org/10.1016/j.ress.2016.04.015

This is a PDF file of an unedited manuscript that has been accepted fo publication. As a service to our customers we are providing this early version o the manuscript. The manuscript will undergo copyediting, typesetting, an review of the resulting galley proof before it is published in its final citable form Please note that during the production process errors may be discovered whic could affect the content, and all legal disclaimers that apply to the journal pertain 


\title{
Optimization of test and maintenance of ageing components consisting of multiple items and addressing effectiveness
}

\author{
I. Martón ${ }^{1 *}$, P. Martorell ${ }^{1}$, R. Mullor ${ }^{3}$, A.I. Sánchez ${ }^{2}$, S. Martorell ${ }^{1}$ \\ ${ }^{1}$ Department of Chemical and Nuclear Engineering. Universitat Politècnica de València, Valencia, Spain \\ ${ }^{2}$ Department of Statistics and Operational Research. Universitat Politècnica de València, Valencia, Spain \\ ${ }^{3}$ Department of Statistics and Operational Research. Universidad de Alicante, Alicante, Spain
}

*Corresponding author: I. Martón (ismarllu@upv.es)

\section{ABSTRACT:}

There are many models in the literature that have been proposed in the last decades aimed at assessing the reliability, availability and maintainability (RAM) of safety equipment, many of them with a focus on their use to assess the risk level of a technological system or to search for appropriate design and/or surveillance and maintenance policies in order to assure that an optimum level of RAM of safety systems is kept during all the plant operational life. This paper proposes a new approach for RAM modelling that accounts for equipment ageing and maintenance and testing effectiveness of equipment consisting of multiple items in an integrated manner. This model is then used to perform the simultaneous optimization of testing and maintenance for ageing equipment consisting of multiple items. An example of application is provided, which considers a simplified High Pressure Injection System (HPIS) of a typical Power Water Reactor (PWR). Basically, this system consists of motor driven pumps (MDP) and motor operated valves (MOV), where both types of components consists of two items each. These components present different failure and cause modes and behaviours, and they also undertake complex test and maintenance activities depending on the item involved. The results of the example of application demonstrate that the optimization algorithm provide the best solutions when the optimization problem is formulated and solved considering full flexibility in the implementation of testing and maintenance activities taking part of such an integrated RAM model.

Keywords: Unavailability, costs, multi-objective optimization, ageing, multiple items, imperfect maintenance, testing intervals, maintenance intervals, NPP component. 
$\lambda_{0} \quad$ Baseline failure rate when the item is new

$R P$ Renewal period

$\alpha \quad$ Linear ageing rate

$\beta$ Shape factor

$\gamma \quad$ Characteristic time

$\varepsilon \quad$ Maintenance effectiveness

$\eta \quad$ Test efficiency

$\rho \quad$ Cyclic or per-demand failure probability

$\lambda^{\mathrm{D}} \quad$ Detected fraction of age-dependent stand-by failure rate

$\lambda^{\mathrm{U}} \quad$ Undetected fraction of age-dependent stand-by failure rate

$\lambda^{\mathrm{UD}}$ Undetected fraction of age-dependent stand-by failure rate that is then detected

$\lambda^{\mathrm{UU}} \quad$ Undetected fraction of age-dependent stand-by failure rate that remains undetected (section 2.1.2)

TI Surveillance test interval

RI Functional test interval

L Life of the item

$\tau \quad$ Downtime for testing,

$\sigma \quad$ Downtime for preventive maintenance,

M Preventive maintenance interval

$\mu \quad$ Downtime for repair

$\Gamma \quad$ Downtime for replacement

$\mathrm{u}^{\mathrm{D}} \quad$ Unavailability due to detected failures

$\mathrm{u}^{\mathrm{UD}}$ Unavailability due to undetected failures by surveillance testing than are then detected by functional tests

$\mathrm{u}^{\mathrm{UU}}$ Unavailability due to undetected failures by both surveillance and functional tests

$\mathrm{u}^{\mathrm{T}} \quad$ Unavailability due to testing

$\mathrm{u}^{\mathrm{M}} \quad$ Unavailability due to performing preventive maintenance

$\mathrm{u}^{\mathrm{C}} \quad$ Unavailability due to performing corrective maintenance

$\mathrm{u}^{\mathrm{O}} \quad$ Unavailability due to replacement of the item

$c_{t} \quad$ Cost contribution as a consequence of performing testing

$\mathrm{c}_{\mathrm{m}}$ Cost contribution as a consequence of performing preventive maintenance

$c_{c}$ Cost contribution as consequence of performing corrective maintenance

$\mathrm{c}_{\mathrm{o}} \quad$ Cost contribution associated with replacing 


\section{INTRODUCTION}

\section{ACCEPTED MANUSCRIPT}

Many models have been developed in the last decades to assess the reliability, availability and maintainability (RAM) of safety equipment. RAM models are developed mainly to assess the risk level of technological systems and/or with a focus on their use to search for appropriate design and/or surveillance and maintenance policies in order to assure that an optimum level of RAM of safety systems is kept during all the plant operational life [1-3].

Complexity of RAM modelling has evolved along this years in an attempt to capture the equipment behaviour in a more realistic way. For example, most of RAM models that were integrated into standard Probabilistic Safety Assessment (PSA), which is the most used tool for safety and risk management in Nuclear Power Plants (NPPs), do not addresses explicitly neither the effect of equipment ageing nor effectiveness of maintenance and testing programs, which could have a significant impact on the conclusions drawn from PSA studies and applications, particularly when NPP are operated at an advanced age or during long term operation. The reason is that equipment ageing and maintenance effectiveness would most likely result in large uncertainty of current component unreliability and unavailability models that support standard PSA quantification, particularly for aged equipment. Fortunately, these effects are limited often implicitly by adopting a living PSA or at least updating the standard PSA regularly, which is mandatory by current regulation in many countries.

In recent years more attention has been paid on modelling explicitly how equipment ageing impacts RAM of safety components and systems. For example, analytical age-dependent unavailability models have been developed adopting linear ageing rates [1], which considers the impact of testing and maintenance activities at least implicitly [2-5]. Nowadays, one can find several proposals in the literature to integrate such a kind of RAM modelling into the so called Ageing PSA (APSA) [6-10].

These studies propose RAM of components should be modelled as a function of the inherent reliability of the component, i.e. component failure rate imposed by design, the component ageing, which degrades the inherent reliability, and the effectiveness of test and maintenance activities, which improve the reliability degraded by ageing, i.e. there is an attempt to return component reliability back to its inherent value eventually, but normally impossible, in case of perfect maintenance activities. In particular, Ref. [6] demonstrates the importance of addressing explicitly the effectiveness of maintenance in managing equipment ageing and test efficiency in detecting hidden failures as this may impact the accurate planning and optimization of testing and maintenance activities based on RAM criteria. This is even more important when the component consists of multiple items, where every item may undertake specific maintenance and testing activities to cope with different degradation mechanisms and failure causes respectively affecting the multiitems component.

On the other hand, one can find in the literature an important number of works devoted to the optimization of test and maintenance intervals of safety systems at NPPs, for example using Genetic Algorithms (GAs), 
which that face either single-objective or multi-objectives adopting RAM plus costs as objectives and/or constraints functions [11-21, 25-30]. Some of them consider the impact of component ageing in the optimization of test and/or maintenance intervals [16-21]. Only several of them considers both test and maintenance interval optimization simultaneously $[12,14,18,20]$.

This paper proposes a new approach for RAM modelling that simultaneously accounts for equipment ageing and maintenance effectiveness and testing efficiency for equipment consisting of multiple items. This model is then used to face the problem of the multi-objective and simultaneous optimization of testing and maintenance intervals for ageing equipment consisting of multiple items. Resolution of such a problem bring a good chance to look for the best balance between component/system availability and cost of the resulting optimal test and maintenance intervals of multiple items components as it is shown in the example of application provided in this paper. The example of application considers a simplified High Pressure Injection System (HPIS) of a typical Pressurized Water Reactor (PWR). Basically, this system consists of ten components: three motor driven pumps (MDP) and seven motor operated valves (MOV). Both component types, MOV and MDP, consist of two items: the motor and pump in the case of the MDP and the actuator and valve in the case of MOV, which are treated separately. These components present different failure modes and behaviours, and also they undertake complex test and maintenance activities, i.e. multiple and different tasks, which depend on the particular item involved.

\section{RAM+C MODELS}

\subsection{Model of a single item}

\subsubsection{Age-dependent failure rate model incorporating imperfect maintenance}

As proposed in [6], the integration of testing and maintenance effectiveness was addressed in an improved APSA. For application, specific ageing models for the failure rate need to be used in the equations for the unavailability and cost models to obtain specific numerical results which can be used to analyze the influence of ageing, imperfect maintenance and testing.

So, the effect of maintenance on the age of the item and on its reliability is included based on a model of imperfect maintenance. Imperfect maintenance models consider that each maintenance activity reduces the age of the item by some degree, depending on its effectiveness. Among the different models of imperfect maintenance that can be found in the literature, this paper considers the Proportional Age Reduction (PAR) model and the Proportional Age Setback (PAS) model proposed in Refs. [2, 3]. The selection of the most appropriate model in each case depends on the item type, failure mechanism and sort of maintenance activity.

In the PAR approach, each maintenance activity is assumed to reduce proportionally the item age gained from the previous maintenance. However, PAS model considers that the maintenance activity reduces proportionally, in a factor of $\varepsilon$, the age that the item has immediately before it enters maintenance. In these models, the effect of maintenance is introduced by using an effectiveness parameter, $\varepsilon$, ranging in the 
interval $[0,1]$. If $\varepsilon=0$, the previous models simply are reduced to an "As Bad as Old" model (normally corrective maintenance), while if $\varepsilon \triangleq 1$ are reduced to an "As Good as New" model (normally overhaul maintenance). For preventive maintenance, $\varepsilon$ ranges in interval $] 0,1[$.

In the literature, one can find different component reliability models proposed to address the effect of equipment ageing, such as linear model, Exponential, Weibull, etc [1].

In this work, two reliability models, Weilbull and Linear, and the imperfect maintenance models presented above, PAS and PAR, are considered to model the age-dependent failure rate. Weibull distribution is widely used in reliability and life data analysis to represent equipment ageing with time due to its versatility [3]. Linear distribution is the simplest way to develop an age-dependent reliability model, which assumes that the failure rate has a linear behaviour with component age departing form an initial value. This assumption, when applicable, simplifies the modelling. In addition, one can find in the literature ageing factors proposed for several components in Nuclear Power Plants [1,9].

According to [2,3], considering a linear ageing model and imperfect maintenance models can be obtained an averaged standby failure rate, $\lambda^{*}$, over the item's life based on a double averaging process [3] which is given by:

$$
\begin{array}{ll}
\lambda^{*}=\lambda_{0}+\frac{\alpha}{2} \cdot M \cdot \frac{(2-\varepsilon)}{\varepsilon} & \text { PAS model } \\
\lambda^{*}=\lambda_{0}+\frac{1}{2} \cdot \alpha \cdot M \cdot\left[1+(1-\varepsilon) \cdot\left(\frac{R P}{M}-1\right)\right] & \text { PAR model }
\end{array}
$$

where, $\lambda_{0}$ is the baseline failure rate when the item is new, $M$ is the maintenance interval, $R P$ is the renewal period and $\alpha$ is the linear ageing rate.

According to [3], considering a Weibull ageing model and PAR or PAS model can be obtained an averaged standby failure rate, $\lambda^{*}$, over the item's life based on a double averaging process [24] which is given by:

$$
\begin{array}{ll}
\lambda^{*}=\lambda_{0}+\frac{M^{\beta-1}}{(\varepsilon \cdot \gamma)^{\beta}} \cdot\left(1-(1-\varepsilon)^{\beta}\right) & \text { PAS model } \\
\lambda^{*}=\lambda_{0}+\frac{(M \cdot \varepsilon+2 \cdot R P \cdot(1-\varepsilon))^{\beta}-(M \cdot \varepsilon)^{\beta}}{R P \cdot(1-\varepsilon) \cdot(2 \cdot \gamma)^{\beta}} & \text { PAR model }
\end{array}
$$

where, $\beta$ and $\gamma$ are the shape and scale factors, respectively. Note that the Weibull distribution simplifies to the linear one for shape factor equal to 2 and the scale factor equal to $\sqrt{2 / \alpha}$. 
2.1.2 Age-dependent failure rate model addressing test efficiency

By testing, component failures can be detected that may have occurred since the last test or the time when the component was last known to be operational. The main objective of surveillance test is to detect hidden failures so that the component can be restored to its operational state. For example, as proposed in Ref. [19], it can be seen like a testing coverage of failure mechanisms, where coverage is defined as a share of detected an undetected failures by testing. Alternatively, Ref. [10] defines test efficiency like the probability that a given failure is detected by the test.

In both previous cases, test efficiency can be represented by a single parameter $\eta$. Ref. [9] and [10] give values of $\eta$ for test efficiency of several component types. As a result, the consideration of a test efficiency splits the total age-dependent failure rate into two age-dependent failure rate modes: detected and undetected.

$$
\lambda^{*}=\lambda^{D}+\lambda^{U}
$$

where the test efficiency, $\eta$, ranges in the interval $[0,1]$. In eqn. (5), the first contribution represents the agedependent failure rate associated with detected failures by testing, $\lambda^{D}$, and the second part represents the age-dependent failure rate associated with undetected failures by testing, $\lambda^{U}$, which can be derived using the corresponding formulation for $\lambda^{*}$ using eqn. (1) to eqn. (4).

$$
\begin{aligned}
& \lambda^{D}=\eta \cdot \lambda^{*} \\
& \lambda^{U}=(1-\eta) \cdot \lambda^{*}
\end{aligned}
$$

On the other hand, a large number of critical components overtakes functional tests mostly performed during refuelling of NPP, where the refuelling Interval (RI) ranges between 12 and 24 months, so that typically RI could be set equal to 18 months. The functional test often involves testing full performance of the component capacity, so that it performs very close to real conditions in case of emergency. Then, the efficiency of such a functional test should be very close to one in detecting hidden failures. Similarly to the surveillance tests, in reliability terminology, the functional test intervals (often adopting the RI) are called BAO intervals since the component age coming out of the test is basically the same as the component age going into the functional test, i.e. the component is as old with regard to its age.

Thus, critical items for NPP safety may overtake at least two tests: one surveillance test and another functional test. Consequently, to address such a second or refuelling functional test, the undetected agedependent failure rate, given by eqn. (7), should split into two new contributions: detected and undetected after the refuelling functional test, to yield

$\lambda^{U D}=\eta_{R I} \cdot \lambda^{U}$

$\lambda^{U U}=\left(1-\eta_{R I}\right) \cdot \lambda^{U}$ 
where the test efficiency of the refuelling functional test, $\eta_{\mathrm{RI}}$, ranges also in the interval $[0,1]$, but very close to one now. In addition, eqn. (8) represents the age-depended failure rate contribution associated with detected failures only after the refuelling functional test, while eqn. (9) represents the age-dependent failure rate contribution associated with failures that remain undetected even after the refuelling functional test.

The formulation proposed in eqns. (5) to (9) can accommodate a number of assumptions made in each application considered.

\subsubsection{Unavailability models}

The unavailability contributions of a single item normally in stand-by are divided into two categories: a) unavailability due to failures, i.e. unreliability effect, and b) unavailability due to testing and maintenance downtimes, named the downtime effect.

a) Unreliability contributions

Adopting the basis of the formulation of unreliability contributions in Ref. [6] considering the contributions introduced in sections 2.1.1 and 2.1.2, the item unavailability due to unreliability contributions can be evaluated using the following equations :

$u^{D} \approx \frac{1}{2} \lambda^{D} \cdot T I$

$u^{U D} \approx \frac{1}{2} \lambda^{U D} \cdot R I$

$u^{U U} \approx \rho+\frac{1}{2} \lambda^{U U} \cdot L$

where $u^{D}$ is the unreliability contribution due to detected failures by surveillance testing, $u^{\text {UD }}$ is the unreliability contribution due to undetected failures by surveillance testing that are then detected by a second functional tests and $\mathrm{u}^{\mathrm{UU}}$ is the unreliability contribution due to undetected failures by both surveillance and functional tests . In addition, the following notation has been used:

$\rho=$ cyclic or per-demand failure probability,

$\lambda^{\mathrm{D}}=$ detected fraction of age-dependent stand-by failure rate (section 2.1.2),

$\lambda^{\mathrm{UD}}=$ undetected fraction of age-dependent stand-by failure rate that is then detected (section 2.1.2),

$\lambda^{\mathrm{UU}}=$ undetected fraction of age-dependent stand-by failure rate that remains undetected (section 2.1.2),

$\mathrm{TI}=$ surveillance test interval,

$R I=$ functional test interval,

$\mathrm{L}=$ life of the item. 
What concerns parameter $\mathrm{L}$, it represents the item life as compared to the NPP design life. In case the item is not replaced by a new one over the NPP design life, then this parameter should be equal to the NPP design life. In case the item undertakes only time-directed overhaul maintenance, this parameter L should be equal to a constant replacement period.

b) Downtime contributions

Based on Ref. [6] addressing the age-dependent failure rate contribution corresponding to detected and repaired failures with the plant at power, as introduced in sections 2.1.1 and 2.1.2, the item unavailability due to detected downtimes for testing and maintenance contributions can be evaluated using the following equations:

$u^{T} \approx \frac{\tau}{T I}$

$u^{M} \approx \frac{\sigma}{M}$

$u^{C} \approx \lambda^{D} \cdot \mu$

$u^{O}=\frac{\Gamma}{L}$

Where, $\mathrm{u}^{\mathrm{T}}$ represents the unavailability contribution due to testing, $\mathrm{u}^{\mathrm{M}}$ is the unavailability contribution due to performing preventive maintenance, $\mathrm{u}^{\mathrm{C}}$ is the unavailability contribution due to performing corrective maintenance, and $\mathrm{u}^{\mathrm{O}}$ is the contribution due to replacement of the item, if any. In addition, the following new notation has been used:

$\tau=$ downtime for testing,

$\sigma=$ downtime for preventive maintenance,

$\mathrm{M}=$ the period to perform time-directed preventive maintenance,

$\mu=$ downtime for repair when there are no time limitations on conducting such a repair,

$\Gamma=$ downtime for replacement.

\subsubsection{Cost models}

The total cost used in the optimization problem considered in this paper include the contributions to the cost model of standby components, related with testing, corrective maintenance to restore the operability after a sudden failure and the failures discovered during test and the cost of preventive maintenance. The cost model for a single item can be evaluated using the following expressions [4]:

$c_{t}=8760 \cdot \frac{c_{t}}{T}$

$\mathrm{c}_{\mathrm{m}}=8760 \cdot \frac{\mathrm{c}_{\mathrm{m}}}{\mathrm{M}}$ 
$c_{c}=8760 \cdot \frac{1}{T} \cdot\left(\rho+\lambda^{D} \cdot T\right) \cdot \mu \cdot c_{c}$

$c_{o}=8760 \cdot \frac{c_{o}}{R P}$

where $c_{t}$ represents a yearly cost contribution as a consequence of the number the test performed on the single item over a year period, $\mathrm{c}_{\mathrm{m}}$ represents a yearly cost contribution as a consequence of performing preventive maintenance on the single item over a year period, $\mathrm{c}_{\mathrm{c}}$ represents a yearly cost contribution as consequence of performing corrective maintenance, and $c_{o}$ is the yearly cost contribution associated with replacing the item with a periodicity RP.

2.2 Model of a system consisting of components with multiple items

\subsubsection{Unavailability model}

The unavailability model of a system which consists of component with multiple $\mathrm{U}(\mathrm{x})$, can be evaluated by using the Fault Tree analysis. The solution of a Fault tree involves the determination of the Minimal Cut Sets (MCS), which represent the unique combinations of component items failure that can cause the system failure. Using the MCS, the system unavailability can be estimated as:

$U(x) \approx \sum_{j} \prod_{k} u_{j k}(x)$

where the sum in $\mathrm{j}$ extents to the number of MCS and the product in k extents to the number of basic events relevant to the corresponding MCS .In addition, $u_{j k}$ represents the unavailability associated with the basic event $\mathrm{k}$ belonging to the MCS number $\mathrm{j}$, which can be evaluated using eqn. (10) to eqn. (16).

\subsubsection{Cost model}

The system total cost system, $\mathrm{C}(\mathrm{x})$, is obtained by summing the cost contributions of the different component items, thus is:

$$
C(x) \approx \sum_{n} \sum_{i} c_{i}(x)
$$

where, $c_{i}$ is the costs associated with the different cost contributors given by eqn. (17) to eqn. (20) for each component item, $\mathrm{n}$, of the system.

\subsection{Parameters estimation}

Different imperfect maintenance models, as shown in previous sections, have been proposed to simulate the impact of maintenance activities on equipment reliability characteristics and, therefore, on unavailability and cost. Each of these models depend on a different set of parameters (effectiveness maintenance, failure rate ...) that can be unknown or uncertain. So, the estimation of these parameters is a council task in modelling the equipment behavior. Usually, the estimation of those parameters is performed for "Bad as old" and "Good as 
new" models. So, it is difficult to find works in the literature focused on maintenance effectiveness and reliability parameters estimation simultaneously [22,23].ANUSCRIPT

In this paper, the estimation of the failure process, such as ageing factor and the maintenance effectiveness have been used to adopt in each case the ageing failure rate model (Linear or Weibull) or the imperfect maintenance model (PAS and PAR). In order to perform this parameters estimation the methodology proposed in [24] has been used which is based on the Maximum Likehood Estimation (MLE). The maximum likelihood estimation (MLE) approach provides estimators, called maximum likelihood estimators, of the parameters involved in the imperfect maintenance models presented in previous section. Maximum likelihood estimates of parameters are those values which make the likelihood function as large as possible, that is, that maximize the probability of the observed data. In this paper the Nelder-Mead Simplex (NMS) method [31] is used to maximize the likelihood function.

\section{OPTIMIZATION PROBLEM}

As said before, the objective of this study is to obtain the optimal test and maintenance intervals for a given safety system under unavailability and cost criteria by means of formulating and solving a multiple objective optimization problem (MOP). In general, MOP includes a set of parameters (decision variables), a set of objective functions and a set of constraints. Objective functions and constraints are defined in terms of the decision variables. The optimization goal can be formulated to minimize a multi-objetive function of the form:

$\mathbf{y}=f(\mathbf{x})=\left(f_{1}(\mathbf{x}), f_{2}(\mathbf{x}), \ldots, f_{n}(\mathbf{x})\right)$

subjected to the vector of constraints

$g(\mathbf{x})=\left(g_{1}(\mathbf{x}) \leq 0, g_{2}(\mathbf{x}) \leq 0, \ldots, g_{n}(\mathbf{x}) \leq 0\right)$

where $\mathrm{x}$ is the decision vector and $\mathrm{y}$ is the objective vector.

In particular, for the problem analyzed in this paper, the frequency of surveillance test and/or maintenance task act as decision variables in vector $\mathrm{x}$, and the objective and constraints vectors are given by:

$\mathbf{y}=f(\mathbf{x})=(U(\mathbf{x}), C(\mathbf{x}))$

$\mathbf{y}=f(\mathbf{x})=\left(U(\mathbf{x}) \leq U_{i}, C(\mathbf{x}) \leq C_{i}\right)$

where $\mathrm{U}(\mathrm{x})$ is the system unavailability given by eqn. (21) and $\mathrm{C}(\mathrm{x})$ is the system cost that is evaluated considering eqn. (22).

The optimization problem has been solved using the fast elitist non-dominated Sorting Genetic Algorithm (NSGA-II) [32]. NSGA-II is the second version of the "Non-dominated Sorting Genetic Algorithm", which solves non-convex and non-smooth single and multi-objective optimization problems. It is an upgrade version 
of its antecessor and its features are: all the individual solutions are sorted according to the level of nondomination, it implements elitism/ convergence properties and it adapts a suitable automatic mechanics based on the crowding distance in order to guarantee diversity and spread of solutions. 


\section{APPLICATION CASE}

\section{ACCEPTED MANUSCRIPT}

The example of application considers a simplified High-Pressure Injection System (HPIS) of a 3-loops Pressurized Water Reactor (PWR) [25]. HPIS is normally in stand-by and consists of three motor driven pumps (MDP) and seven motor operated valves (MOV) organized as shown in Figure 1. Note, there may be different configurations in other plants. Under accidental conditions, the HPIS can be used to remove heat from the Reactor Coolant System in those events in which steam generators are unavailable. For example, in case of a Small-Break Loss-Of-Coolant Accident the HPIS safety function draws water from the Refueling Water Storage Tank (RWST) and discharges it into the cold legs of the Reactor Cooling System through any of the two injection paths. Normally, MDP discharge into the injection paths A and B through MOV 3 and 5, although crossover MOV 4, 6 and 7 provide alternative flow paths in case of failure of the normal feed.

Figure. 1. High Pressure Injection (HPIS) system

Motor-operated valves and motor-driven pumps consist of two items each, which are treated separately, the motor and pump in the case of the MDP and actuator and valve in the case of MOV [24, 25]. These components present different failure and cause modes and behaviours, and also they undertake complex test and maintenance activities, i.e. multiple and different tasks, which depend on the particular item involved. Figures 2 and 3 provide an overview of the relationships considered between components, items, failure and cause modes, surveillance tests and maintenance activities.

Figure 2. Overview of basic interactions for MDP

Figure 3. Overview of basic interactions for MOV

Reliability and maintainability models and parameters were fitted using the methodology proposed in [24, 25] and the operational data and maintenance history, i.e. maintenance effectiveness $(\varepsilon)$, imperfect maintenance model (PAS/PAR), per-demand failure probability $(\rho)$, stand-by failure rate $\left(\lambda_{0}\right)$, ageing model (Linear/Weibull) and ageing factor $(\alpha, \beta, \gamma)$. The results obtained after the estimation process are summarized in Table 1, which shows the most appropriate reliability and maintenance simulation models.

Table 1. Models and parameters obtained in the estimation process 
In addition, values of test efficiency of both surveillance and functional test were taken from NUREG/CR5587 [10]. As said, test efficiency can be formulated in terms of the percentage of the total failure rate that is detected by the test. Note that TI represents a surveillance test while RI represents a functional test, which almost always is performed during refueling period, being the last one most effective in detecting failures. Both tests have associated an effectiveness, $\eta$ for surveillance tests and $\eta_{R I}$ for functional test. Other parameters like M, L, maintenance durations (corrective, preventive and overhaul maintenance), test durations and test intervals, which have been taken from NPP data, are shown in Table 2.

Table 3 shows the unitary costs per activity and component-item used to calculate the several cost contributions, which have been taken from Ref. [2].

Table 2. Data of tests and maintenance for MDP and MOV

Table 3. Component item cost parameters

The objective of this paper is focused on performing a multi-objective optimization under cost and unavailability criteria of testing and maintenance intervals of the multi-item MDPs and MOVs of the HPIS system, considering equipment ageing, maintenance effectiveness and test efficiency in an integrated manner.

In this optimization process, only surveillance tests (TI) are considered as decision variable because RI is fixed to a constant value equal to 13140 hours, which represents the functional test performed every refueling period (18 months) of the NPP. Typical values of TI are established within the Technical Specifications of the NPP. In particular, Techs Specs of the HPIS establish a value of TI=2184 hours for both MDP and MOV, as it is shown in Table 2. In addition, Techs Specs of NPP use to establish the development of surveillance test adopting either a sequential or staggered test strategy. Then, every component is not tested as stand-alone component, instead of this, several components are tested under the same test strategy, so that, they must share the same test periodicity. Accordingly, Table 4 shows surveillance tests of the ten components of the HPIS have been grouped into three test strategies $\{$ TI1, TI2, TI3 $\}$, which represents that all components in the same group share the same TI.

On the other hand, what concerns scheduling of maintenance activities as decision variables, Table 4 shows four groups of maintenance intervals for the HPIS system. The first two $\{M 1, M 2\}$ apply to all MOV of the system, where M1 refers to maintenance activities of the mechanical part, the valve, while M2 refers to maintenance activities of the electrical part, the actuator. Similarly, $\{$ M3, M4 $\}$ apply to all MDP of the system, where M3 refers to the mechanical part, the pump, and M4 to the electrical part, the motor. It is assumed initially that all preventive maintenance strategies are performed with the same maintenance interval, M, equal to 4320 hours. This assumption will change depending on the optimization case as described in the following. 


\section{ACCEPTED MANUSCRIPT}

Table 4. Decision variables: Test and Maintenance Intervals.

Multi-Objective optimization under cost and unavailability criteria has been performed using the nondominated Sorting Genetic Algorithm (NSGA-II) [32] toolbox in Matlab using eqn. (25) as objective function. Relevant parameters used in the optimization process are showed in Table 5.

Table 5. NSGA-II parameters

Six optimization cases has been developed adopting, in part or as a whole, the set of decision variables presented in Table 4. Lower and upper bounds have been adopted for the decision variables, which allows searching for solutions in the desired domain of feasible ones. Thus, $\mathrm{TI}_{\mathrm{i}}(\mathrm{i}=1,3)$ must range in the interval $[24,80000]$ while $M_{j}(j=1,4)$ must range in the interval [720, 80000]. As said, the initial surveillance test and maintenance intervals are $\mathrm{TI}_{\mathrm{i}}=2184$ and $\mathrm{M}_{\mathrm{j}}=4320(\forall \mathrm{i}=1,3$ and $\mathrm{j}=1,4)$.

Table 6 shows the six optimization problems that have been formulated and solved. All cases adopts the same objective function consisting of both unavailability and costs for the HPIS, which are calculated using reliability and maintenance data showed in Tables 1 to 3 , and the initial or optimized values for the decision variables in Table 4 depending on the particular case. The NSGA-II algorithm with the same parameters shown in Table 5 is used for all six cases.

Table 6. Cases studied

Cases 1 to 3 adopt test efficiency equal to one. This means unavailability of each component resets to the residual value, normally zero, after the surveillance test, i.e. the surveillance test is able to detect whatever hidden failure of the component and therefore it is assumed the component is available after the test provided that the component is not found failed at the test. Cases 4 to 6 considers a test efficiency for each MDP and MOV equal to 0.6 and 0.4, respectively, which are taken from NUREG 5587 [10]. In addition, Case 1 and 4 address surveillance test interval (TI) optimization only. Case 2 and 5 address optimization of both surveillance test intervals (TI) and maintenance intervals (M). Case 3 and 6 address are similar to the previous one. However, now maintenance is grouped either by electrical or by mechanical maintenance, considering that maintenance for each group share the same period. Therefore, now M1 is set equal to M3 and M2 is set equal to M4.

Figure 4 and Figure 5 show the results of the optimization process. Figure 4 shows the Pareto Fronts corresponding to Cases 1 to 3. Figure 5 shows the Pareto Fronts corresponding to Cases 4 to 6 . 
Figure 4 shows that better results are obtained when the whole set of TI and M are considered in the decision making of the optimization problem (Cases 2 and 3) as compared to the worst results found for the case where only the set of TI is optimized (Cases 1). The value of unavailability and cost obtained are significantly lower in Cases 2 and 3. In addition, better results are found under Case 2 as compared to Case 3 in terms of optimal unavailability and cost for the HPIS. This means that the best results can be found by including the whole set of decision variables and allowing flexibility in the decision making. Note that in Case 3 maintenance activities are grouped under electrical and mechanical types only and therefore the decision space is reduced. Similar results can be found in Figure 5.

On the other hand, by comparing Figures 4 and 5, one can realize the importance of surveillance test efficiency, since the unavailability of the HPIS is underestimated in all cases represented in Figure 4. In addition, when a test efficiency lower than one is considered, which is a more realistic assumption based on data available, there is no so big difference between grouping or not maintenance activities into electrical and mechanical types, see Cases 5 and 6 in Figure 5 as compared to their corresponding Cases 2 and 3 in Figure 4.

Figure 4. Pareto Front obtained for Cases 1 to 3

Figure 5. Pareto Front obtained for Cases 4 to 6

\section{CONCLUDING REMARKS}

Many approaches have been suggested in the past to optimize the surveillance test and maintenance intervals of a system based on various analytical unavailability and cost models. Most of these approaches do not consider simultaneously and explicitly the impact of equipment ageing, maintenance effectiveness and surveillance testing efficiency, in particular for components consisting of multiple items.

This paper proposes a new approach for RAM modelling that simultaneously accounts for equipment ageing and maintenance effectiveness and testing efficiency for equipment consisting of multiple items. This model is then used to face the problem of the multi-objective and simultaneous optimization of testing and maintenance intervals for ageing equipment consisting of multiple items.

The results obtained in the application case show substantial differences, mainly in the assessment of the HPIS system unavailability, when ageing and both maintenance effectiveness and testing efficiency are considered explicitly. Thus, the example of application shows that maintenance effectiveness in managing ageing and test efficiency in detecting hidden failures have a significant impact on the results found, so that, future research should be directed towards the estimation, or even improvement, of maintenance effectiveness and test efficiency and the assessment of their effect on failure causes and failure modes affecting RAM of safety equipment, since they impact the accurate planning and optimization of testing and maintenance activities based on RAM criteria. 
The results of the example of application demonstrate that the optimization algorithm provide the best solutions when the optimization problem is formulated and Csolved considering full flexibility in the implementation of testing and maintenance activities taking part of such an integrated RAM model of multiple items components. Thus, the multi-objective optimization problem provide a Pareto front of solutions corresponding with different values of the decision variables (test and maintenance). Each one of the set of optimal (and different) combination of test and maintenance intervals corresponds to one realization of the Pareto front, which provides balance between system unavailability and total cost. In NPP operation, decision maker would select the most appropriate combination of above optimized set that corresponds to the necessary balance between unavailability and cost.

\section{REFERENCES}

[1] Vesely W.E., Kurth R.E., Scalzo S.M. Evaluations of core melt frequency effects due to component ageing and maintenance. NUREG/CR-5510. Washington. DC: USNRC, 1990.

[2] Sanchez A., Carlos S., Martorell S., Villanueva J.F. Addressing imperfect maintenance modelling uncertainty in unavailability and cost based optimization. Reliability Engineering and System Safety 2009; 94 (1): 22-32.

[3] Martorell S., Sanchez A., Serradell V. Age-dependent reliability model considering effects of maintenance and working conditions. Reliability Engineering and System Safety 1999; 64: 19-31.

[4] Martorell, S., Villanueva, J. F., Carlos, S., Nebot, Y., Sánchez, A., Pitarch J. L. and Serradell, V. RAMS+C informed decision-making with application to multi-objective optimization of technical specifications and maintenance using genetic algorithms. Reliability Engineering \& System Safety 2005; 87(1):65-75.

[5] Kancev D., Cepin M. (2011). Evaluation of risk and cost using an age-dependent unavailability modelling of test and maintenance for standby components. Journal of Loss Prevention in the Process Industries 2011, 24 (2); 146-155

[6] Martón I., Sánchez A.I., Martorell S., Ageing PSA incorporating effectiveness of maintenance and testing, Reliability Engineering \& System Safety 139: 131-140, 2015

[7] Volkanovski A. Method for assessment of ageing based on PSA results, Nuclear Engineering and Design 2012; 246: 141146.

[8] European Comission, JRC Science and Policy Reports, Feasibility Study for applicability of Ageing PSA model results in Risk-informed Decision Process, APSA Network, 2014

[9] Levy. I. et al S., Wreathall. J., DeMoss., G..Wolford. A. J. Collins. E. P., \& Jarrell. D. B. (1988). Prioritization of TIRGALEX recommended components for further ageing research. NUREG/CR-5248. Washington. DC: US NRC.

[10] U.S. Nuclear Regulatory Commission. Approaches for Age-Dependent Probabilistic Safety Assessments with Emphasis on Prioritization and Sensitivity Studies. NUREG/CR-5587. SAIC-92/1137. Washington DC, 1992.

[11] Cepin, M. (2002). Optimization of safety equipment outages improves safety. Reliability Engineering \& System Safety, 77, 71-80.

[12] Courtois, P.-J., \& Delsarte, P. (2006). On the optimal scheduling of periodic tests and maintenance for reliable redundant components. Reliability Engineering \& System Safety, 91, 66-72

[13] Martorell, S., Carlos, S., Sánchez, A., \& Serradell, V. (2000). Constrained optimization of test intervals using a steady-state genetic algorithm. Reliability Engineering \& System Safety, 67, 215-232.

[14] Martorell, S., Carlos, S., Villanueva, J. F., Sanchez, A. I., Galvan, B., Salazar, D., et al. (2006). Use of multiple objective evolutionary algorithms in optimizing surveillance requirements. Reliability Engineering \& System Safety, 91, 1027-1038 
[15] Volkanovski, A., Cepin, M., \& Mavko, B. (2009). Nuclear power plant maintenance optimization. In R. Bris (Ed.), Reliability, risk and safety: theory and applications. Proceedings and Monographs in Engineering, Water and Earth Sciences, ESREL 2009, Vol. 3. Prague, Czech Republic: Taylor \& Francis. 2010, pp. 2151-2147.

[16] Kancev D., Gjorgiev B., Cepin M. (2011). Optimization of test interval for ageing equipment: A multi-objective genetic algorithm approach. Journal of Loss Prevention in the Process Industries 24: 397-404

[17] Martorell, S., Sánchez, A., Carlos, S., \& Serradell, V. (2002a). Comparing effectiveness and efficiency in technical specifications and maintenance optimization. Reliability Engineering \& System Safety, 77, 281e289.

[18] Martorell, S., Sánchez, A., Carlos, S., \& Serradell, V. (2002b). Simultaneous and multicriteria optimization of TS requirements and maintenance at NPPs. Annals of Nuclear Energy, 29, 147-168.

[19] Mishra, A., Patwardhan, A., \& Verma, A. K. (2007). Safety management in NPPs using an evolutionary algorithm technique. Nuclear Engineering and Design, 237, 1445-1451.

[20] Munõz, A., Martorell, S., \& Serradell, V. (1997). Genetic algorithms in optimizing surveillance and maintenance of components. Reliability Engineering \& System Safety, 57, 107-120.

[21] Martorell, S., Sanchez, A., \& Serradell, V. (1999). Age-dependent reliability model considering effects of maintenance and working conditions. Reliability Engineering \& System Safety, 64, 19-31.

[22] Shin, I., Lim T.J. \& Lie, C.H. (1996). Estimating parameters of intensity function and maintenance effect for repairable unit. Reliability Engineering and Systems Safety 54 1-10.

[23] Cheng, C.Y. \& Liaw, F. (2005). Statistical estimation on imperfectly maintained system. Advances in safety and reliability. Proceedings ESREL 2005. 351-356.

[24] Mullor R. (2006). Parameters estimation under preventive imperfect maintenance”. ESREL 2006 Safety and Reliability for Managing Risk, Estoril (Portugal), Setember 2006.

[25] Mullor R. (2007). Motor-operated valve maintenance optimization considering multiple failure modes and imperfect maintenance models”. ESREL 2007 Safety and Reliability for Managing Risk, Stavanger (Norway), June 2007.

[26] Ayoobian, N., Mohsendokht, M. (2016), Multi-objective optimization of maintenance programs in nuclear power plants using Genetic Algorithm and Sensitivity Index decision making annals of nuclear energy,88, 95-99.

[27] Compare, M.; Martini, F.; Zio, E. (2015), Genetic algorithms for condition-based maintenance optimization under uncertainty, European journal of operational research, 244(2): 611-623.

[28] Coria, V. H.; Maximov, S.; Rivas-Davalos, F.; et al. (2015), Analytical method for optimization of maintenance policy based on available system failure data, reliability engineering \& system safety, 135:55-63.

[29] Khataba, A., Aghezzafb, E.H., 2016, Selective maintenance optimization when quality of imperfect maintenance actions are stochastic Reliability Engineering \& System Safety,150: 182-189.

[30] Abdul H., Faisal K., , Salim A., (2016), A risk-based shutdown inspection and maintenance interval estimation considering human error, Process Safety and Environmental Protection, 100: 9-21.

[31] Nelder, J.A.\& Mead,R. (1965).ASimplex method for function minimization. Computer Journal, 308-313

[32] Deb, K., PrataP, A., Agarwal, S., Meyarivan, T., A fast and elitist multiobjective genetic algorihtm. NSAG-II. IEEE Transactions on Evolutionary Computation, 6(2), 182-197, 2002.

\section{ACKNOWLEDGMENTS}

Authors are grateful to the Spanish Ministry of Science and Innovation for the financial support of this work (Research Project ENE2013-45540-R) and the doctoral fellow (BES-2011-043906) and (BES-2014-067602). 


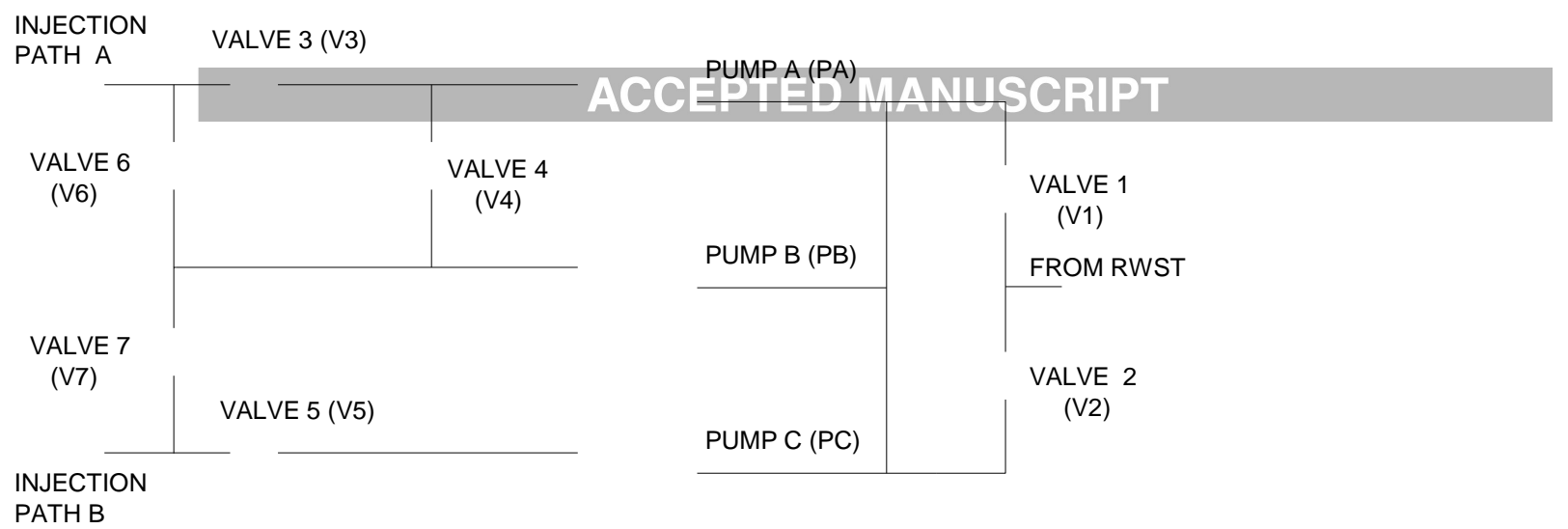

Figure. 1. High Pressure Injection (HPIS) system

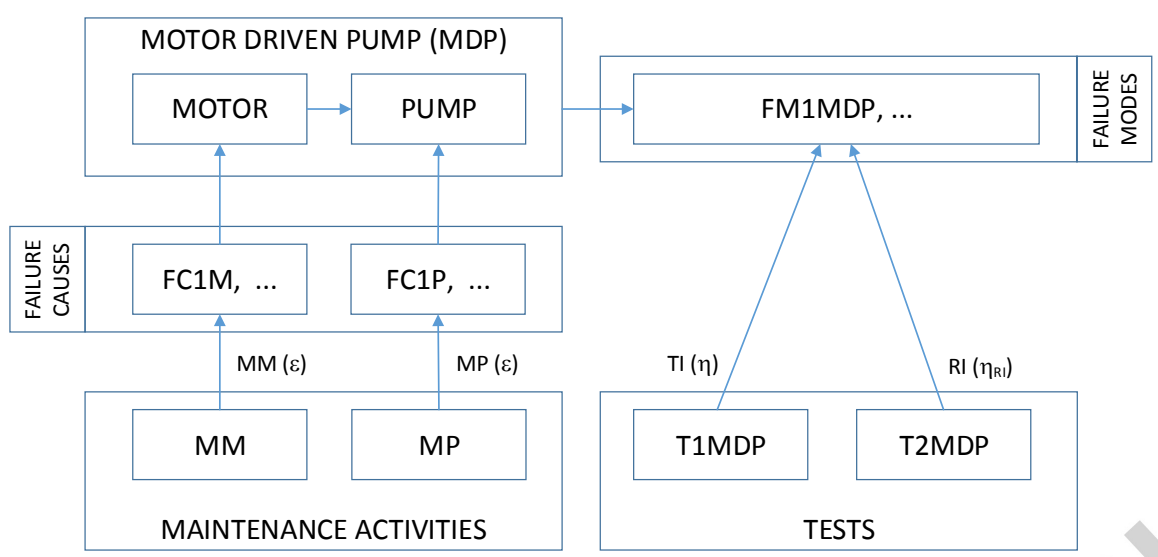

Figure 2. Overview of basic interactions for MDP

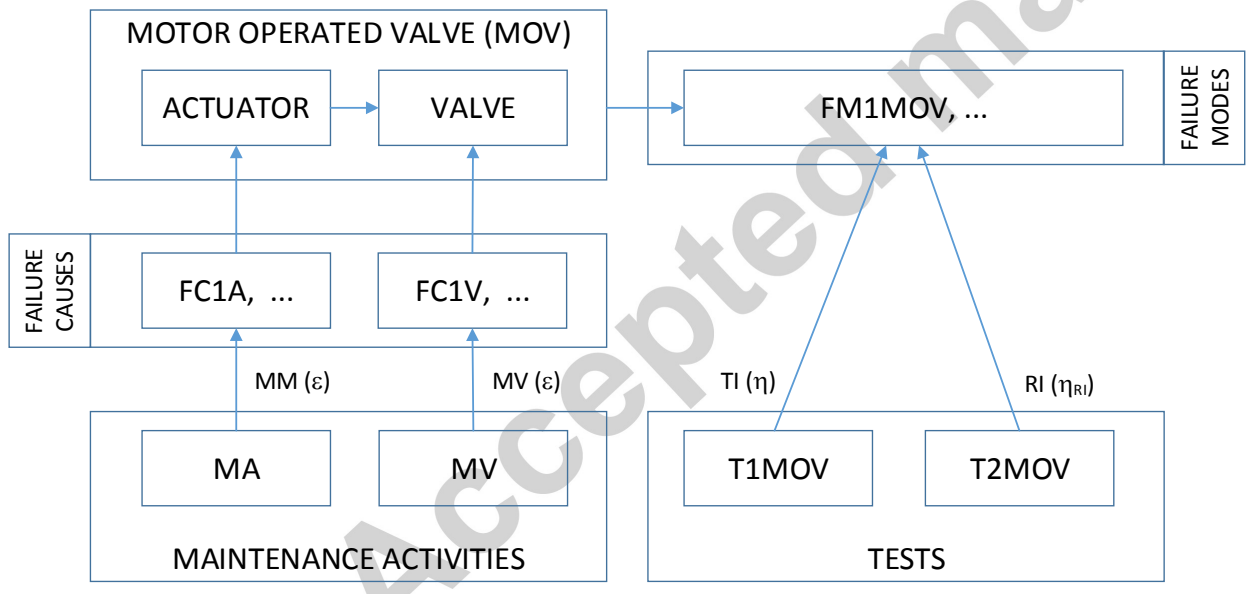

Figure 3. Overview of basic interactions for MOV 


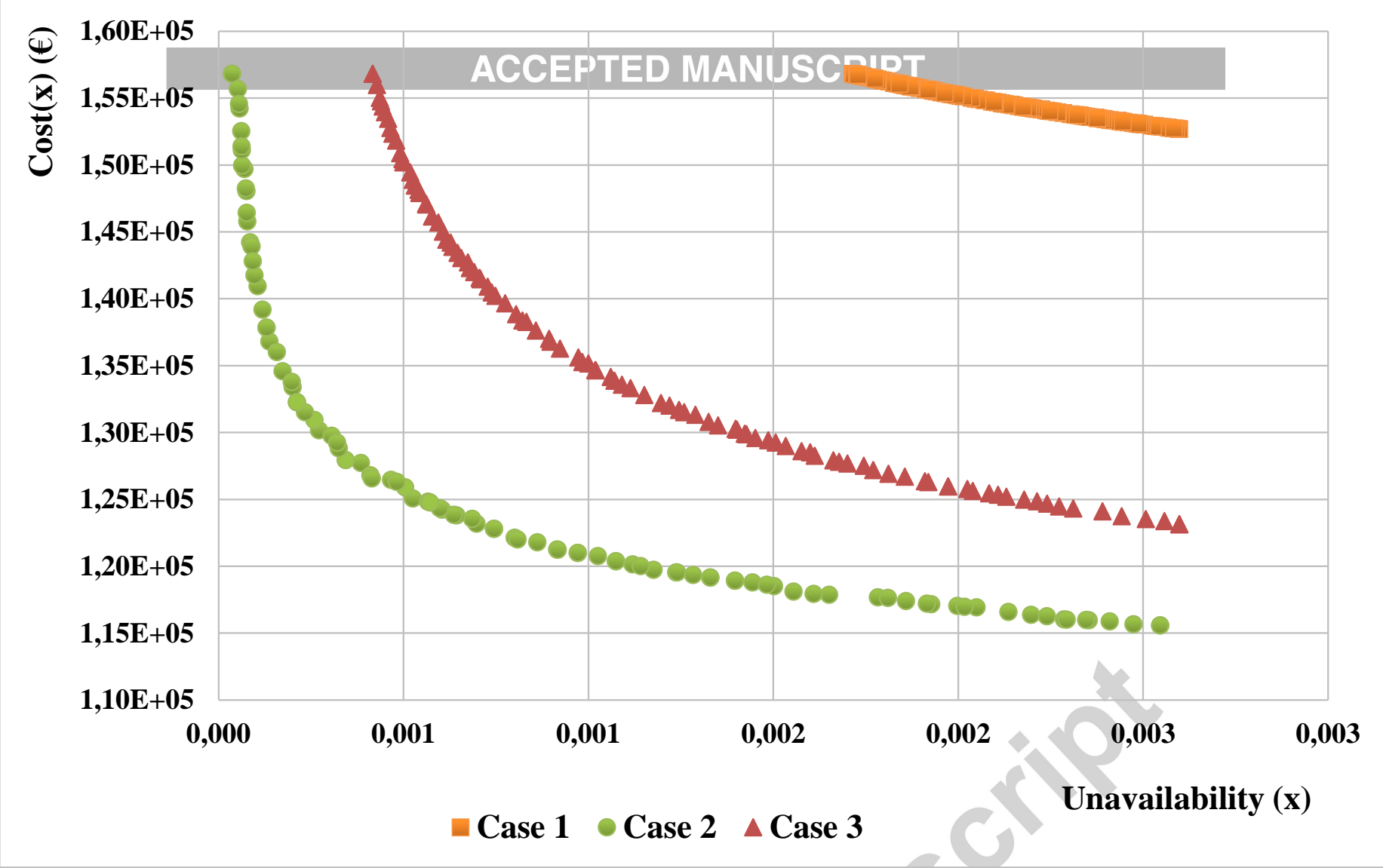

Figure 4. Pareto Front obtained for Cases 1 to 3

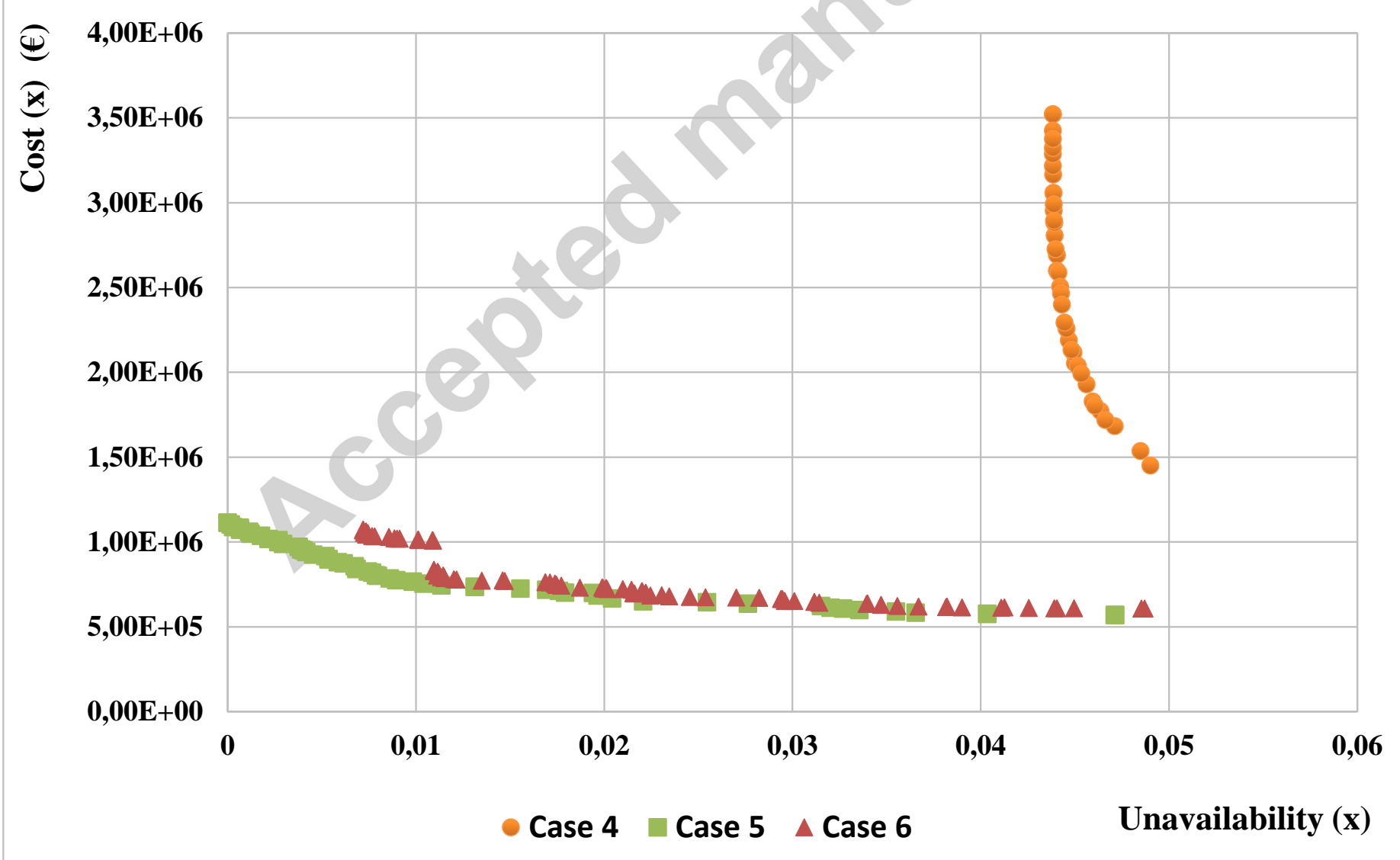

Figure 5. Pareto Front obtained for Cases 4 to 6

Table 1. Models and parameters obtained in the estimation process 


\begin{tabular}{|c|c|c|c|c|c|}
\hline & \multicolumn{2}{|c|}{ Motor operated valve (MOV) } & \multicolumn{2}{|c|}{ Motor driven pump (MDP) } & \multirow[b]{2}{*}{ Units } \\
\hline & Valve & Actuator & Pump & Motor & \\
\hline$\rho$ & $1.81 \mathrm{E}-03$ & 1.25E-05 & 5.18E-04 & $1.25 \mathrm{E}-05$ & {$[-]$} \\
\hline$\lambda_{0}$ & $6.80 \mathrm{E}-06$ & 7.00E-06 & 2.30E-05 & 3.89E-06 & {$[1 / \mathrm{h}]$} \\
\hline IM model & PAR & PAS & PAR & PAS & -- \\
\hline$\varepsilon$ & 0.76 & 0.84 & 0.77 & 0.29 & -- \\
\hline Ageing Model & Linear & Weibull & Linear & Weibull & -- \\
\hline$\alpha$ & $1.73 \mathrm{E}-09$ & -- & 2.37E-09 & -- & {$\left[\mathrm{h}^{-2}\right]$} \\
\hline$\beta$ & -- & 4.87 & -- & 7.47 & -- \\
\hline$\gamma$ & -- & 33347 & -- & 15397 & -- \\
\hline
\end{tabular}

Table 2. Data of tests and maintenance for MDP and MOV

\begin{tabular}{lccccc}
\hline & \multicolumn{2}{l}{ Motor operated valve (MOV) } & \multicolumn{2}{l}{ Motor driven pump (MDP) } & \\
& Valve & Actuator & Pump & Motor & Units \\
\hline$\sigma$ & 1 & 1 & 10 & 10 & {$[\mathrm{~h}]$} \\
$\mathrm{TI}$ & 2184 & 2184 & 2184 & 2184 & {$[\mathrm{~h}]$} \\
$\eta$ & $\{1,0.6\}$ & 13140 & $11,0.44\}$ & $\{1,0.44\}$ & -- \\
$\mathrm{RI}$ & 13140 & 1 & 13140 & 13140 & {$[\mathrm{~h}]$} \\
$\eta_{\mathrm{RI}}$ & 1 & 1 & 1 & 1 & -- \\
$\tau$ & 1 & 2,6 & 4 & 4 & {$[\mathrm{~h}]$} \\
$\mu$ & 2,6 & 6 & 24 & 24 & {$[\mathrm{~h}]$} \\
$\Gamma$ & 6 & 122640 & 122640 & 50 & {$[\mathrm{~h}]$} \\
$\mathrm{L}$ & 122640 & & & & {$[\mathrm{~h}]$} \\
\hline
\end{tabular}

Table 3. Component item cost parameters

\begin{tabular}{lccccc}
\hline & Units & Valve & Actuator & Pump & Motor \\
\hline$c_{\mathrm{t}}$ & {$[€ /$ year] } & 400 & 400 & 100 & 100 \\
$c_{\mathrm{c}}$ & {$[€ /$ year] } & 25920 & 2880 & 2808 & 312 \\
$c_{\mathrm{m}}$ & {$[€ /$ year] } & 7200 & 800 & 720 & 80 \\
$c_{\mathrm{o}}$ & {$[€ /$ year] } & 32400 & 3600 & 3240 & 360 \\
\hline
\end{tabular}

Table 4. Decision variables: Test and Maintenance Intervals.

Interval Component Item Type of




\begin{tabular}{lllll}
\hline Maintenance strategy & M1 CCEV1, V2,V3,V4,V5,V6,V7 & Valve & Mechanical \\
& M2 & V1, V2,V3,V4,V5,V6,V7 & Actuator & Electrical \\
M3 & PA, PB, PC & Pump & Mechanical \\
Testing strategy & M4 & PA, PB, PC & Motor & Electrical \\
& TI1 & V1, V2 & -- & Surveillance Test \\
& TI2 & V3, V5, PA, PB, PC & -- & Surveillance Test \\
& TI3 & V4, V6, V7 & -- & SurveillanceTest \\
\hline
\end{tabular}

Table 5. NSGA-II parameters

\begin{tabular}{ll}
\hline Parameter & Value \\
\hline Generations & 1000 \\
Population Size & 100 \\
Crossover rate & $2 / 7$ \\
Crossover type & Arithmetic Crossover \\
Mutation rate & $2 / 7$ \\
Mutation type & Gaussian Mutation \\
Stopping criteria & Maximum generations number \\
\hline
\end{tabular}

Table 6. Cases studied

\begin{tabular}{lll}
\hline $\begin{array}{ll}\text { Case \# } \\
\text { Test Effectiveness } \\
\text { Case } 1\{1,1\}\end{array}$ & Description & Decision variables \\
\hline Case $2\{1,1\}$ & TI optimization & $\{\mathrm{TI} 1, \mathrm{TI} 2, \mathrm{TI} 3\}$ \\
\hline Case $3\{1,1\}$ & TI and M optimization & $\{\mathrm{TI} 1, \mathrm{TI} 2, \mathrm{TI} 3, \mathrm{M} 1, \mathrm{M} 2, \mathrm{M} 3, \mathrm{M} 4\}$ \\
& $\begin{array}{l}\text { TI and M optimization } \\
\text { Maintenance grouped by type of } \\
\end{array}$ & $\begin{array}{l}\{\mathrm{TI} 1, \mathrm{TI} 2, \mathrm{TI}, \mathrm{M} 1, \mathrm{M} 2, \mathrm{M} 3, \mathrm{M} 4\} \\
\text { being: }\end{array}$ \\
& & $\mathrm{M} 1=\mathrm{M} 3$ \\
\hline Case $4\{0.6,0.4\}$ & TI optimization & $\mathrm{M} 2=\mathrm{M} 4$ \\
\hline Case $5\{0.6,0.4\}$ & TI and M optimization & $\{\mathrm{TI} 1, \mathrm{TI} 2, \mathrm{TI} 3\}$ \\
\hline Case $6\{0.6,0.4\}$ & TI and M optimization & $\{\mathrm{TI}, \mathrm{TI} 2, \mathrm{TI} 3, \mathrm{M} 1, \mathrm{M} 2, \mathrm{M} 3, \mathrm{M} 4\}$ \\
& Maintenance grouped by type of & $\{\mathrm{TI} 1, \mathrm{TI} 2, \mathrm{TI} 3, \mathrm{M} 1, \mathrm{M} 2, \mathrm{M} 3, \mathrm{M} 4\}$ \\
& maintenance: electrical or mechanical & B1=M3 \\
& & M2=M4 \\
\hline
\end{tabular}

Highlights

New approach for RAM modelling

Equipment ageing and maintenance effectiveness and testing efficiency explicitly in RAM models

Equipment consisting of multiple items

Multiple objective optimization problem (MOP) under unavailability and cost criteria 
